\title{
A psychoacoustical study to investigate the perceived unpleasantness of infrasound combined with audio-frequency sound
}

\author{
Elisa Burke ${ }^{1, *}$, Stefan Uppenkamp ${ }^{2}$, and Christian Koch ${ }^{1}$ \\ ${ }^{1}$ Physikalisch-Technische Bundesanstalt (PTB), 38116 Braunschweig, Germany \\ ${ }^{2}$ Medizinische Physik, Universität Oldenburg, 26111 Oldenburg, Germany
}

Received 20 December 2019, Accepted 12 September 2020

\begin{abstract}
At many immission sites of infrasound (frequency $f<20 \mathrm{~Hz}$ ), humans are exposed to a mixture of infrasound and sound in the common audio-frequency range (audio sound, $20 \mathrm{~Hz}<f<20 \mathrm{kHz}$ ). Therefore, the purpose of this study was to examine the auditory perception of infrasound and audio sound not only in isolation but also in combination. This laboratory study aims to investigate the perceived unpleasantness of infrasound (sinusoid at $12 \mathrm{~Hz}$ ) and audio sound (sinusoid at $1000 \mathrm{~Hz}$, pink-noise 250-4000 Hz), presented alone or in combination with each other. A pairwise comparison task and a rating task using a numerical scale were conducted with 19 normal hearing listeners. In addition, individual detection thresholds were determined for the infrasound stimulus. Combinations of infrasound and audio sound were rated as equally or more unpleasant than either sound presented alone. Inter-individual differences in unpleasantness ratings using the numerical scale were particularly high for stimuli containing infrasound. This can only in part be related to the large variability in infrasound thresholds. These findings suggest that simultaneous exposure to infrasound and audio sound can increase the perceived unpleasantness when both are presented at a sufficient level above the detection threshold.
\end{abstract}

Keywords: Infrasound, Unpleasantness, Psychoacoustic scaling methods, Detection threshold

\section{Introduction}

There is ongoing debate to what extent environmental noise with components in the infrasound frequency range (frequencies $f$ below $20 \mathrm{~Hz}$ ), e.g. noise originating from road and railway traffic, aircrafts, heat pumps, ventilation system or wind turbines, may cause annoyance and possibly affect human health (see e.g. the review papers [1-3]). However, as Berglund et al. [1] pointed out, caution must be taken that most field studies on low frequency noise, including infrasound, investigate effects of "broadband noise with a predominant or significant low-frequency component. Thus, the effects of low-frequency noise per se are difficult to identify" ([1], p. 2996). Moreover, measured sound pressure levels of environmental infrasound in the human environment are often below the perception threshold [1].

On the other hand, several laboratory studies investigated the perception of pure infrasound. Detection threshold measurements demonstrated that humans are able to perceive infrasound when its sound pressure level (SPL) is sufficiently high (see e.g. [4, 5]). In addition, it was found that the distances between equal-loudness-curves $[4,6]$ as well as between equal-annoyance-curves $[7,8]$ decrease with

*Corresponding author: elisa@burkegermany.de decreasing frequencies below $100 \mathrm{~Hz}$, so that the dynamic range of the human auditory system in the infrasonic frequency range is considerably smaller than in the common audio-frequency range ("audio sound", $20 \mathrm{~Hz}<f<$ $20 \mathrm{kHz}$ ). Thus, taking into account the large inter-individual variability in detection thresholds for infrasound [4, 5], an infrasound stimulus which is perceived as very loud for one individual can be still inaudible for someone else. Furthermore, Inukai et al. [9] conducted measurements of equal-unpleasantness curves from $10 \mathrm{~Hz}$ to $500 \mathrm{~Hz}$ in a laboratory setting. They found that acceptable limits for lowfrequency sound were well above threshold-level, but depended on the imagined situation of the sound exposure (e.g. in a bed room, in an office).

A distinction needs to be made between the terms "annoyance" and "unpleasantness" of noise. In general, the term "annoyance" defines a multifactorial psychological concept, covering evaluative aspects, like unpleasantness and nuisance, and behavioural aspects, e.g. interference with intended activities and disturbance [10]. Moreover, personal factors, e.g. noise sensitivity and personality traits, and social factors, e.g. attitudes of the society towards the noise source, moderate the perceived annoyance [11]. Even if the exact meaning of the term "annoyance" varies for different languages [12], the "unpleasantness" is seen as one of the 
main components of the annoyance concept from English, German and Japanese speaking experts [10]. In particular, the term "unpleasantness" is defined by Guski as the "instantaneous evaluation of a situation (e.g. a sound)" ([12], p. 767).

Since human exposure to infrasound is usually accompanied by higher frequencies, i.e. audio sound, it is crucial to investigate whether the interaction of infrasound and audio sound affects the quality of the auditory perception and the perceived annoyance. Only few laboratory studies exist so far that focussed on the perception and annoyance of combined infrasound and audio sound stimuli. Within one experiment of Møller's study [8], participants rated the annoyance of infrasound (pure tone at $16 \mathrm{~Hz}$ ) combined with audio sound (narrowband noise centred at $1000 \mathrm{~Hz}$ ) for different sound pressure levels, after they had been exposed to the stimuli in a pressure chamber with the interfering task of reading newspapers. The study revealed that combinations of infrasound and audio sound were rated as equally or slightly more annoying than either sound presented alone. The psychoacoustical study of Marquardt and Jurado [13] showed that a simultaneous exposure to infrasound and audio sound cannot be distinguished from an exposure to an audio sound that is amplitude modulated at the same infrasonic frequency. This confirmed the finding of Salt et al. [14] whose experiment with cochlea of guinea pigs gave evidence that, when audio sound and infrasound are simultaneously presented to the ear, audio sound may be amplitude modulated at an infrasonic modulation frequency due to physiological mechanisms. In an earlier study, Burke et al. [15] performed monaural detection threshold measurements for combined infrasound and audio sound. It was found that thresholds for infrasound pure tones at $5 \mathrm{~Hz}$ and at $12 \mathrm{~Hz}$ can be significantly increased when simultaneously presented with audio sound, e.g. sinusoid at $100 \mathrm{~Hz}$ and a broadband pink noise stimulus, at a level of $50 \mathrm{~dB}$ SL (sensation level). However, vice versa, no masking effect was found for audio sound thresholds caused by the presence of the same infrasound pure tones presented up to $10 \mathrm{~dB}$ SL.

The study presented in this paper should give a more profound investigation of the unpleasantness, as a precursor of the perceived annoyance, for infrasound combined with audio sound. The experiments were conducted in a laboratory setting to ensure a controlled stimulation with infrasound and audio sound, presented alone or in combination with each other. Moreover, this study focussed on the perception of infrasound and audio sound by means of the auditory system and using airborne stimulation only. All other ways of sensation, e.g. somatosensory mechanisms, are intentionally ruled out for this investigation. Therefore, instead of a whole-body exposure as in Møller's study [8], a monaural stimulation with infrasound and audio sound stimuli was realized by using tubes and an audiometric ear tip to transfer the sound from the sound sources to the listener's ear canal [16]. In addition, the term "unpleasantness" was used rather than "annoyance" since the participants should judge the stimuli without interfering task and should focus on the auditory evaluation only.
The purpose of this study was to compare and to quantify the unpleasantness for combined infrasound and audio sound stimuli, at different sound pressure levels. In particular, this study aimed to investigate whether combinations of infrasound and audio sound were rated as more unpleasant than either sound presented alone. On the one hand, combinations of infrasound and audio sound may be perceived as more unpleasant than the same stimuli presented alone due to the resulting modulation effect $[13,14]$ since laboratory studies found that the annoyance of sounds increases with the depth of periodic amplitude modulation (see e.g. $[17,18])$. On the other hand, combinations of infrasound and audio sound stimuli may be equally unpleasant as audio sound presented alone since the masking effect of audio sound on infrasound thresholds [15] may also affect the perceived unpleasantness. Burke et al. [15] assumed that a non-energetic masking effect is a possible reason for the threshold increase for infrasound caused by audio sound, meaning that the attention of the listener being exposed to infrasound and audio sound might be shifted to the perception of audio sound. Therefore, the presence of audio sound may also reduce the perceived unpleasantness from infrasound, even for infrasound stimuli presented well above the detection threshold. In addition, the effect of the level of infrasound and audio sound stimuli on unpleasantness ratings was examined. Moreover, it was of special interest to investigate whether there is a relationship between individual detection thresholds for infrasound and individual unpleasantness ratings for stimulation with infrasound, possibly combined with audio sound.

\section{Materials and methods}

\subsection{Participants}

Nineteen listeners aged between 18 and 30 years participated in the hearing tests. To reduce a potential sensitisation of the participants to infrasound, none of the listeners who participated in the previous study on detection thresholds for combined infrasound and audio sound stimuli [15] were included in this study. In general, none of the listeners had experience with psychoacoustic experiments with infrasound prior to this study. All of the participants were otologically normal as tested by an otoscopic examination. In addition, the listeners had hearing thresholds better than $15 \mathrm{~dB}$ HL for the right ear for pure tones between $125 \mathrm{~Hz}$ and $8000 \mathrm{~Hz}$ as tested by standard pure tone audiometry according to ISO 8253-1 [19], with a step size of $1 \mathrm{~dB}$. The Declaration of Helsinki was adhered to in all the measurements and a positive vote of the local ethics committee (PTB ethics application 3/16) was given.

\subsection{Outline of this study}

The unpleasantness of combined infrasound and audio sound stimuli was investigated using two different psychoacoustic scaling methods. A paired comparison task (see Sect. 2.5.1) was conducted as the first experiment. This method has already been applied in other studies to 
investigate the auditory unpleasantness of sounds (e.g. [20]). Thereafter, an unpleasantness rating task using an 11-pointnumerical scale (see Sect. 2.5.2) was performed, which is a scale recommend by the International Commission on the Biological Effects of Noise (ICBEN) [21] and by ISO/TS $15666[22]$ to determine noise-induced annoyance.

After the two unpleasantness rating tasks, the individual detection threshold (see Sect. 2.5.3) for the infrasound stimulus at $12 \mathrm{~Hz}$ was determined. The intention of this was to analyse whether there might be a relationship between the individual detection thresholds for infrasound and individual unpleasantness ratings for infrasound, presented alone or in combination with audio sound. The threshold measurement was the last experiment for each participant to avoid a potential sensitisation to infrasound prior to the unpleasantness rating tasks.

The set of the three experiments (unpleasantness ratings tasks and detection threshold measurement) was performed within one day for each participant. The total duration of the three experiments was around $2.5 \mathrm{~h}$ (including breaks).

The preliminary results from the unpleasantness rating tasks for the first six from a total of 19 participants had already been presented during the International Congress on Acoustics (ICA Aachen 2019, see conference paper [23]). Thus, the same methods for the unpleasantness rating tasks were also applied for the remaining 13 participants completing the present study. However, for these 13 listeners, the numerical scale ratings for each stimulus were repeated twice (instead of once only for the first six participants), to gather more robust data. The current study also includes the data for individual infrasound detection thresholds from all 19 participants that had not been presented in the conference proceedings.

Since the experiments were the same for the first six and the remaining 13 participants, except only for the number of repetitions in the unpleasantness rating task using the numerical scale, the data from all listeners were analysed together, assuming the small experimental discrepancy had no influence.

\subsection{Setup}

The infrasound and audio sound stimuli were generated by a specially developed sound source system which is described and characterised in more detail in [16]. In short, the sound source system allows a monaural stimulation for infrasound combined with audio sound at low harmonic distortion. Moreover, the application of a compensation source reduces the generation of unwanted modulation frequencies during the simultaneous presentation of infrasound and audio sound stimuli down to an imperceptible level [16]. A slight modification in the measurement setup compared to the one in [16] was that the time delay unit (see setup in [16]) was removed in this study. It was not needed any more, as only one frequency in the infrasonic frequency range was used in this study.

The infrasound and audio sound signals were digitally generated in MATLAB at $96 \mathrm{kHz}$ sample rate. The digitalto-analogue $(\mathrm{D} / \mathrm{A})$ conversion of the signals was performed with an external sound card (RME Fireface UC, infrasound and audio sound components in separate channels) whose outputs were connected to amplifiers (type Tira BAA 120 for audio signals and type Beak BAA 120 for infrasound signals). The acoustic infrasound and audio sound stimuli were each generated by different electrodynamic loudspeakers mounted in separated housings. Their sound was transferred by a tube system to the same audiometric eartip (E-A-RTone/E-A-RLink, Standard Insert Foam Eartips) that was inserted in the listener's right ear during the hearing test. The contralateral ear of the listener was occluded with an earplug (Honeywell, Bilsom 303 SingleUse Earplug). The listeners and the sound source system were in an anechoic room. The background level of the anechoic room is illustrated in a figure in [15], demonstrating that it is sufficiently below the detection thresholds, even in the infrasonic frequency range. In front of the listener, there was a computer screen, a mouse, and a keyboard, which were used for controlling the experiments. The acoustic output of the sound source system was calibrated with an IEC 60318-4 [24] occluded ear-simulator (Brüel \& Kjær 4157, with ear canal extension DB 2012) for stimuli with frequencies above $100 \mathrm{~Hz}$. The calibration measurements for infrasonic frequencies were conducted with a $\frac{1^{\prime \prime}}{2}$ low-frequency pressure-field microphone (Brüel \& Kjær 4193, with UC0211) coupled to a cavity with a volume of $1.3 \mathrm{~cm}^{3}$, which is approximately equivalent to the typical volume of the human ear canal according to IEC 60318-4 [24].

\subsection{Stimuli}

A set of 26 different stimuli (Table 1) was applied in the unpleasantness rating tasks, including a sinusoid at $12 \mathrm{~Hz}$, a broadband pink-noise stimulus (short: "broadband stimulus", frequency range between $250 \mathrm{~Hz}$ and $4000 \mathrm{~Hz}$ ), and a sinusoid at $1000 \mathrm{~Hz}$, presented alone or in combination with each other at different sound pressure levels. One reason for the choice of these specific stimuli was that they were also applied in the previous study of Burke et al. [15] investigating detection thresholds for combined infrasound and audio sound stimuli. The present study also aims to investigate whether the observed masking of infrasound caused by audio sound [15] may also influence the perceived unpleasantness. The reason for the choice of the specific frequency range of the broadband stimulus was that its centre frequency should correspond to that of the sinusoid, its lower cut-off frequency should be above the infrasonic frequency range, and it was also taken into account that the recommended frequency range of the loudspeaker emitting audio sound was between 50 and $6000 \mathrm{~Hz}$ according to [16]. Furthermore, the two different audio sound stimuli were applied to analyse whether the unpleasantness ratings differ between a broadband stimulus and a sinusoid, presented alone or in combination with infrasound. This is to be expected assuming that tonal sounds are perceived as more unpleasant than non-tonal sounds, which is taken into account in national noise regulations (see, e.g. DIN 45681:2005-03 [25]) by adding a penalty value to the 
Table 1. List of applied stimuli.

\begin{tabular}{lccccc}
\hline \multirow{2}{*}{ No. } & \multicolumn{2}{c}{ Infrasound } & & \multicolumn{2}{c}{ Audio sound } \\
\cline { 2 - 3 } \cline { 5 - 6 } & Stimulus & SPL/dB & & Stimulus & SPL/dB \\
\hline 1 & $12 \mathrm{~Hz}$ & 95 & & - & - \\
2 & $12 \mathrm{~Hz}$ & 110 & & - & - \\
3 & - & - & & Broadband & 21 \\
4 & - & - & & Broadband & 36 \\
5 & - & - & & Broadband & 53 \\
6 & $12 \mathrm{~Hz}$ & 80 & & Broadband & 21 \\
7 & $12 \mathrm{~Hz}$ & 80 & & Broadband & 36 \\
8 & $12 \mathrm{~Hz}$ & 80 & & Broadband & 53 \\
9 & $12 \mathrm{~Hz}$ & 95 & & Broadband & 21 \\
10 & $12 \mathrm{~Hz}$ & 95 & & Broadband & 36 \\
11 & $12 \mathrm{~Hz}$ & 95 & & Broadband & 53 \\
12 & $12 \mathrm{~Hz}$ & 110 & & Broadband & 21 \\
13 & $12 \mathrm{~Hz}$ & 110 & & Broadband & 36 \\
14 & $12 \mathrm{~Hz}$ & 110 & & Broadband & 53 \\
15 & - & - & & $1000 \mathrm{~Hz}$ & 20 \\
16 & - & - & & $1000 \mathrm{~Hz}$ & 50 \\
17 & - & - & & $1000 \mathrm{~Hz}$ & 70 \\
18 & $12 \mathrm{~Hz}$ & 80 & & $1000 \mathrm{~Hz}$ & 20 \\
19 & $12 \mathrm{~Hz}$ & 80 & & $1000 \mathrm{~Hz}$ & 50 \\
20 & $12 \mathrm{~Hz}$ & 80 & & $1000 \mathrm{~Hz}$ & 70 \\
21 & $12 \mathrm{~Hz}$ & 95 & & $1000 \mathrm{~Hz}$ & 20 \\
22 & $12 \mathrm{~Hz}$ & 95 & & $1000 \mathrm{~Hz}$ & 50 \\
23 & $12 \mathrm{~Hz}$ & 95 & & $1000 \mathrm{~Hz}$ & 70 \\
24 & $12 \mathrm{~Hz}$ & 110 & & $1000 \mathrm{~Hz}$ & 20 \\
25 & $12 \mathrm{~Hz}$ & 110 & & $1000 \mathrm{~Hz}$ & 50 \\
26 & $12 \mathrm{~Hz}$ & 110 & & $1000 \mathrm{~Hz}$ & 70 \\
\hline & & & &
\end{tabular}

measured level of noise with tonal components. However, it must also be taken into account that the tone-to-noise ratio, the mutual masking and the absolute loudness of the components affect the perceived annoyance caused by noisetone combinations (see e.g. [26]).

The stimuli were presented at different levels to investigate the effect of the level of infrasound and of audio sound stimuli on the unpleasantness ratings for combined infrasound and audio sound stimuli, and whether there is an interaction between them. The sinusoid at $12 \mathrm{~Hz}$ was presented at $95 \mathrm{~dB}$ SPL and $110 \mathrm{~dB}$ SPL (Table 1, No. 1-2, No. 9-14 and No. 21-26), which represents sound pressure levels around and clearly above the average threshold level reported in [15], that is a sound pressure level around $92 \mathrm{~dB}$. Since sound pressure levels of environmental infrasound noise are often below threshold level (e.g. [1]) it was particularly interesting to investigate whether audio sound combined with infrasound at a level below threshold might be more unpleasant than audio sound in isolation. Therefore, the infrasound stimulus was also presented at a sound pressure level of $80 \mathrm{~dB}$, that is, below detection threshold, when it was combined with audio sound (stimulus No. 6-8 and No. 18-20). The sinusoid at $1000 \mathrm{~Hz}$ was presented at 20, 50 , and $70 \mathrm{~dB}$ SPL. The broadband stimulus was also presented at three sound pressure levels $(21,36$, and $53 \mathrm{~dB}$ SPL) having approximately the same loudness as the sinusoidal stimulus at $1000 \mathrm{~Hz}$ according to [27].

The duration of each stimulus presentation in the unpleasantness ratings tasks (see Sect. 2.5) was set to two seconds, so that the results of both experiments remain comparable. The duration of two second was chosen to keep the total duration of the pairwise comparison task cycle with 650 pairs of stimuli within an acceptable range for the participant (average duration of pairwise comparison task: $70 \mathrm{~min}$ in total without breaks). In the detection threshold measurements, the duration of the stimuli was set to one second, according to the threshold measurement for a sinusoid at $12 \mathrm{~Hz}$ in [15]. All stimuli had a $0.25 \mathrm{~s}$ cosine squared ramp at onset and offset. The broadband stimulus was digitally pre-shaped to equalise the frequency response of the audio sound source. In order to avoid learning effects the (broadband) noise stimulus was freshly generated prior to each presentation using random number creation tools of the software (i.e., "running noise").

\subsection{Psychoacoustic methods}

\subsubsection{Pairwise comparison unpleasantness task}

The participants were asked to compare the unpleasantness for all combinations of the 26 stimuli listed in Table 1. In each measurement trial, two unequal stimuli were presented one after another to the participant. Simultaneous to the acoustic presentation, two panels labelled " 1 " and " 2 " were successively highlighted in red colour on the computer screen in front of the participant. Then, the question "Which stimulus was more unpleasant" (translated from German: "Welcher Stimulus ist unangenehmer?") appeared on the screen and the participants answered the question by clicking on the respective panel. Each pair of two stimuli was compared twice, once as $\mathrm{A}-\mathrm{B}$ and once as $\mathrm{B}-\mathrm{A}$. The presentation order of the total number of 650 paired comparisons was pseudo-randomized for each participant.

The responses of the participant $k=1, \ldots, 19$ to the comparisons of the 26 stimuli were each summarised in a $26 \times 26$-matrix $A_{k}$. The entries $a(i, j)$ of the matrix $A_{k}$ represent the mean result of the two comparisons between the stimulus in the row $i$ (abbr.: "stimulus $i$ ") and the stimulus in the column $j$ (abbr.: "stimulus $j$ ') for all $i \neq j$ and for $i$, $j=1, \ldots, 26$. The entry $a(i, j)$ would equal 1 , if stimulus $i$ was rated as more unpleasant than stimulus $j$ in both comparisons. Vice versa, $a(i, j)$ becomes " 0 ", if stimulus $j$ was rated as more unpleasant in both comparisons. One has $a(i, j)=0.5$ for contrary ratings for the repeated comparisons. The "winning rate" $W R(i)$ was defined as the relative frequency with which a listener rated a particular stimulus $i$ as more unpleasant than the other 25 stimuli. Hence, the winning rate $W R(i)$ of each stimulus $i$ was calculated as the sum of the row entries of $A_{k}$ (for $i \neq j$ ) divided by " 25 " that is the number of the comparisons for each stimulus:

$$
W R(i)=\frac{\sum_{j=1, i \neq j}^{26} a(i, j)}{25} .
$$

Then, the matrices $A_{k}$ with the unpleasantness ratings of the 19 participants were summed up to one matrix $B$ with 
entries $b(i, j)=0, \ldots, 19$, for $i, j=1, \ldots, 26$ and for $i \neq j$. Based on the data of the paired comparison task, the following questions were investigated: (1) Are combinations of infrasound and audio sound stimuli more unpleasant than either stimulus presented alone (30 comparisons)? (2) Is infrasound presented alone more unpleasant than audio sound presented alone (twelve comparisons)? (3) Does the unpleasantness of the isolated stimuli increase with an increase in level (seven comparisons)? (4) Does the unpleasantness of the broadband stimulus differ from that of the $1000-\mathrm{Hz}$ sinusoid at the same loudness level when they are presented alone or in combination with infrasound (twelve comparisons)? A statistical analysis was conducted to test the significance for each of these 61 selected comparisons (of a total of 325 , that is $13 \times 25$ comparisons for 26 sounds) whether one stimulus was more or less unpleasant than the other. More specifically, the hypothesis was tested that the unpleasantness responses of the participants for one particular comparison of two stimuli $b(i, j)$ were not just by chance. The first step of this analysis was to simulate the frequency distribution of the summed responses $b$ $(i, j)$ which would have resulted when all participants repeatedly would have given responses at random (for one million iterations). The assumptions for the simulated distribution were that the four possible responses of each participant were uniformly distributed and independent of each other. The four responses were: $(1) a(i, j)=1$ (see above), (2) $a(i, j)=0$ (see above), (3) $a(i, j)=0.5$ : first stimulus $i$ was rated as more unpleasant, then stimulus $j$ was rated as more unpleasant, $(4) a(i, j)=0.5$ : reverse order of response (3). Based on the simulated distribution, the critical values $b_{\text {crit,lowerlimit }}(i, j)$ and $b_{\text {crit,upperlimit }}(i, j)$ could be calculated by applying a significance level $\alpha=0.05 / 61$ for a two tailed test (Bonferroni corrected for 61 tested comparisons). The critical values indicate a stimulus $i$ that was significantly either more unpleasant $\left(b(i, j)>b_{\text {crit,upperlimit }}\right.$ $(i, j)=14.5)$ than stimulus $j$ or less unpleasant than stimulus $j\left(b(i, j)<b_{\text {crit,lowerlimit }}(i, j)=4.5\right)$.

\subsubsection{Unpleasantness rating task using an 11-point numerical scale}

In this unpleasantness rating task, the participant rated the unpleasantness of each of the 26 stimuli (listed in Table 1) by means of an 11-point numerical scale. The participants were instructed prior to the experiments that they should judge the unpleasantness of each stimulus, even if it is possible that they did not hear a sound. A field labelled "sound" was highlighted in red colour on the screen in front of the participants while a stimulus was presented. After the acoustic presentation, the participants were asked to indicate the unpleasantness of the stimulus by choosing a number between "0" labelled "not at all unpleasant" (translated from German: "überhaupt nicht unangenehm") to "10" labelled "extremely unpleasant" (translated from German: "extrem unangenehm"). The unpleasantness ratings were repeated for each stimulus. One repetition was performed for the first six participants. To increase the robustness of the results, two repetitions were performed for the
Table 2. The factors values, investigated effects and $p$-values of ART-ANOVA 1 are listed. Significant effects and significant interactions $(p<0.05)$ are indicated by an asterisk. The factor value "low level (2)" of the factor 'level of audio sound' includes the unpleasantness ratings averaged over the ratings for the sinusoid at $1000 \mathrm{~Hz}$ at $20 \mathrm{~dB}$ SPL and for the broadband at $21 \mathrm{~dB}$ SPL. In addition, the factor value "medium level (3)" includes the unpleasantness ratings averaged over the ratings for the sinusoid at $1000 \mathrm{~Hz}$ at $50 \mathrm{~dB}$ SPL and for the broadband at $36 \mathrm{~dB}$ SPL, and the factor value "high level (4)" includes the averaged ratings for the sinusoid at $1000 \mathrm{~Hz}$ at $70 \mathrm{~dB}$ SPL and for the broadband at $53 \mathrm{~dB}$ SPL, respectively.

\section{ART-ANOVA 1}

\begin{tabular}{lc}
\hline Factor 1: Level of audio sound & Factor 2: Level of infrasound \\
\hline No audio sound (1) & $95 \mathrm{~dB}$ SPL (1) \\
Low level (2) & $110 \mathrm{~dB} \mathrm{SPL} \mathrm{(2)}$ \\
Medium level (3) & \\
High level (4) & $p$-value \\
Investigated effects & $p<0.0001^{*}$ \\
\hline Factor 1 & $p<0.0001^{*}$ \\
Factor 2 & $p<0.0001^{*}$ \\
Factor 1:Factor 2 & \\
\hline
\end{tabular}

remaining 13 participants. The presentation order of the total number of 52 or 78 ratings was pseudo-randomized. The participants were instructed prior to this experiment that the same stimuli as in the first experiment (paired comparison task) were presented in order to reduce the risk of sequence effects (i.e. participants evaluate each stimulus by internal comparison with the previously presented stimuli) at the beginning of the experiment.

The data of the numerical rating task were analysed with the Aligned Rank Transform Analyses of Variance (ART-ANOVA) [28] using the $\mathrm{R}$ package ARTool [29]. The ART-ANOVA is a non-parametric analysis which is suitable for testing main effects and interactions in a multifactorial design [28]. Analyses of variance (ANOVAs) were performed with the aligned and ranked data to investigate effects and interactions of the different audio sound stimuli (pink noise and sinusoid at $1000 \mathrm{~Hz}$ ) and the levels of both, infrasound and audio sound stimuli, on the unpleasantness ratings. Since the design of our study was not full factorial, e.g. "no infrasound" was not used in combination with "no audio sound", the analysis of the data was separated in two ART-ANOVAs to achieve a balanced design of the models. The factor values of the ART-ANOVAs are summarised in Tables 2 and 3.

\subsubsection{Detection threshold measurement}

Detection threshold levels for a sinusoid at $12 \mathrm{~Hz}$ were determined three times for each participant, using an adaptive 1-up-2-down 3-alternative forced-choice (3-AFC) procedure. The start level of the stimulus was $105 \mathrm{~dB}$ SPL, that is above the average detection threshold level according to [15]. The initial step size was $4 \mathrm{~dB}$. The step size was reduced to $3 \mathrm{~dB}$ and to $2 \mathrm{~dB}$ after the first and second 
Table 3. The factors values, investigated effects and $p$-values of ART-ANOVA 2 are listed. Significant effects and significant interactions $(p<0.05)$ are indicated by an asterisk. The factor level "low level (1)" of the factor 'level of audio sound' corresponds to the unpleasantness rating of the sinusoid at $1000 \mathrm{~Hz}$ at $20 \mathrm{~dB}$ SPL or of the broadband at $21 \mathrm{~dB}$ SPL. In addition, the factor value "medium level (2)" corresponds to the unpleasantness rating of the sinusoid at $1000 \mathrm{~Hz}$ at $50 \mathrm{~dB}$ SPL or of the broadband at $36 \mathrm{~dB}$ SPL, and the factor level "high level (3)" corresponds to the unpleasantness rating of the sinusoid at $1000 \mathrm{~Hz}$ at $70 \mathrm{~dB}$ SPL or of the broadband at $53 \mathrm{~dB}$ SPL.

\begin{tabular}{lcc}
\hline & ART-ANOVA 2 & \\
\hline Factor 1: Type of audio sound & Factor 2: Level of audio sound & Factor 3: Level of infrasound \\
\hline Broadband (1) & Low level (1) & No infrasound (1) \\
Sinusoid at 1000 Hz (2) & Medium level (2) & 80 dB SPL (2) \\
& High level (3) SPL (3) & $110 \mathrm{~dB}$ SPL (4) \\
& & $p$-value \\
Investigated effects & & $p<0.0001^{*}$ \\
\hline Factor 1 & & $p<0.0001^{*}$ \\
Factor 2 & $p<0.0001^{*}$ \\
Factor 3 & $p=0.0003^{*}$ \\
Factor 1:Factor 2 & $p<0.0001^{*}$ \\
Factor 1:Factor 3 & $p<0.0001^{*}$ \\
Factor 2:Factor 3 & $p=0.6933$ \\
Factor 1:Factor 2:Factor 3 & & $p=0$
\end{tabular}
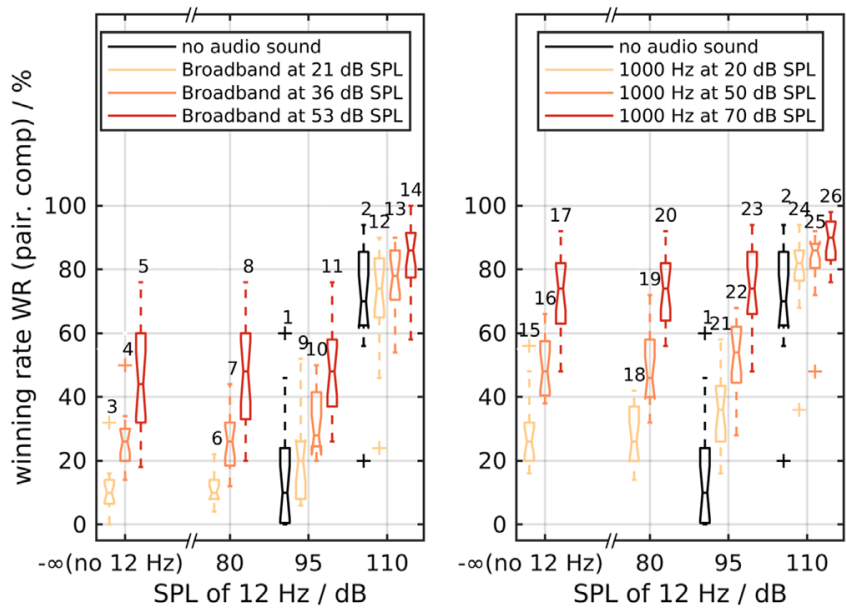

Figure 1. Boxplots of average unpleasantness ratings of 19 participants based on a pairwise comparison test for different stimulus combinations of infrasound and audio sound. The number above each boxplot refers to the stimulus number in Table 1 .

upper reversal. The measurement phase began after the second lower reversal and ended upon the completion of the eighth following reversal. The experiment was implemented with the MATLAB-based software framework AFC [30].

The threshold level was calculated as the median value of the eight reversals during the measurement phase. Correlations (Spearman's rank correlation coefficient) were calculated between the individual infrasound thresholds and individual unpleasantness ratings for each stimulus. Average thresholds were calculated as the arithmetic mean across all participants and associated standard deviations of the means with $68 \%$ confidence interval.
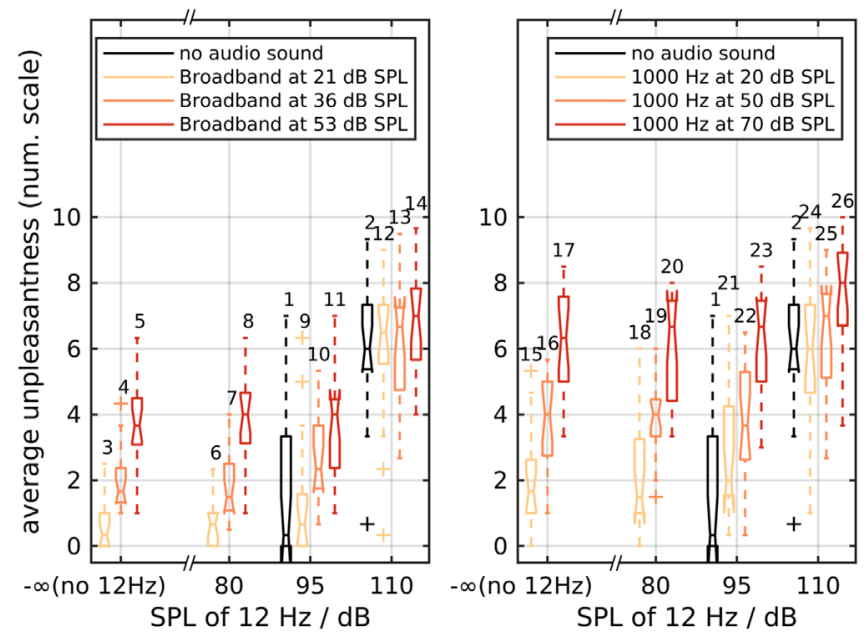

Figure 2. Boxplots of average unpleasantness ratings of 19 participants using an 11-point numerical scale for different stimulus combinations of infrasound and audio sound. The number above each boxplot refers to the stimulus number in Table 1.

\section{Results}

\subsection{Paired comparison task}

Figure 1 shows the boxplots of the winning rate (calculated according to Sect. 2.5.1) for all participants. The whiskers cover the range of data points that are located inside 1.5 times the interquartile range above the upper quartile and below the lower quartile. Data points located outside these limits are marked as outliers by a plus sign. The colour of the boxplot indicates the level of the audio sound stimulus and the position of the boxplot on the $x$-axis indicates the level of the infrasound stimulus. The number 
of each stimulus which refers to the stimulus number listed in Table 1 is inserted above each boxplot.

Figure 1 shows that the sinusoid at $12 \mathrm{~Hz}$ presented at $110 \mathrm{~dB}$ SPL and the sinusoid at $1000 \mathrm{~Hz}$ presented at $70 \mathrm{~dB}$ SPL, alone or in combination with another stimulus, were rated as the most unpleasant stimuli with median winning rates $W R$ greater than or equal to $70 \%$. Winning rates for the isolated $12 \mathrm{~Hz}$ stimulus exhibit a wide spread of values (range of values: $0-60 \%$ for $12 \mathrm{~Hz}$ at $95 \mathrm{~dB}$ SPL and 20 $94 \%$ for $12 \mathrm{~Hz}$ at $110 \mathrm{~dB}$ SPL).

Statistical tests were conducted to test for specific comparisons whether one stimulus was rated significantly more unpleasant than another stimulus across the participants (see Sect. 2.5.1). The results of the tests showed that the unpleasantness for isolated audio sound (broadband and sinusoid at $1000 \mathrm{~Hz}$ ) and isolated infrasound (sinusoid at $12 \mathrm{~Hz}$ ) increased with increasing SPL, which can also be seen in Figure 1 as an increase in winning rate with increasing SPL. In addition, the isolated $12 \mathrm{~Hz}$ stimulus at $95 \mathrm{~dB}$ SPL was equally unpleasant as the isolated audio sound stimuli at the lowest level (broadband at $21 \mathrm{~dB}$ SPL, and $1000 \mathrm{~Hz}$ at $20 \mathrm{~dB}$ SPL), and it was less unpleasant than the audio sound stimuli at the medium and highest level (broadband at 36 and $53 \mathrm{~dB} \mathrm{SPL}$, and $1000 \mathrm{~Hz}$ at 50 and $70 \mathrm{~dB} \mathrm{SPL}$ ). On the contrary, the isolated $12 \mathrm{~Hz}$ at the higher level of $110 \mathrm{~dB}$ SPL was rated as more unpleasant than the isolated audio stimuli, except for the isolated sinusoid at $1000 \mathrm{~Hz}$ at $70 \mathrm{~dB}$ SPL which was rated as equally unpleasant.

Audio sound combined with subthreshold infrasound $(12 \mathrm{~Hz}$ at $80 \mathrm{~dB} \mathrm{SPL})$ revealed the same unpleasantness as the same isolated audio sound stimuli. All combinations of audio sound with the $12 \mathrm{~Hz}$ stimulus at $95 \mathrm{~dB}$ SPL were rated as equally unpleasant as the isolated audio sound stimuli at the same level. The combination of the $12 \mathrm{~Hz}$ stimulus at $95 \mathrm{~dB}$ SPL with the broadband stimulus at $21 \mathrm{~dB}$ SPL or at $36 \mathrm{~dB}$ SPL was rated as equally unpleasant as the same infrasound stimulus presented alone. However, all remaining combinations of infrasound at $95 \mathrm{~dB}$ SPL and audio sound were rated as more unpleasant than the isolated infrasound stimulus at $95 \mathrm{~dB}$ SPL. The audio sound stimuli combined with the $12 \mathrm{~Hz}$ stimulus at $110 \mathrm{~dB}$ SPL showed the same unpleasantness as the same infrasound stimulus presented alone, except for the combination of $1000 \mathrm{~Hz}$ stimulus at $70 \mathrm{~dB}$ SPL with the high-level infrasound which was rated as more unpleasant than the isolated infrasound. Moreover, the combinations of audio sound stimuli with the high-level infrasound were more unpleasant than the same audio sound stimuli presented alone.

The isolated sinusoid at $1000 \mathrm{~Hz}$ was rated as more unpleasant than the isolated broadband noise at the same loudness level, except for the highest level they were equally unpleasant. This also applies if the audio sound stimuli were each presented simultaneously with the sinusoid at $12 \mathrm{~Hz}$ at $80 \mathrm{~dB}$ SPL and at $95 \mathrm{~dB}$ SPL, except for the comparison of stimulus No. 22 and 10 which were rated as equally unpleasant.

\subsection{Numerical scale ratings}

The individual results of the unpleasantness ratings are shown as boxplots in Figure 2 arranged in the same way as the results in Figure 1. The individual ratings were averaged over the two (for the first six participants) or three (for the remaining 13 participants) repeated measurements for each stimulus. The intra-individual variability calculated as the maximum difference between the lowest and highest unpleasantness rating for each stimulus across the two or three repetitions showed that the individual unpleasantness responses were consistent: The intra-individual variability across all stimuli yielded a median of "1" for 17 from 19 participants and a value of "0" or "2" for the remaining two participants. In addition, the interquartile ranges of the intraindividual variability across stimuli were between " 0 " and "3" for all participants. A closer inspection of individual results revealed inter-individual differences, which were especially high for unpleasant ratings of $12 \mathrm{~Hz}$ at $95 \mathrm{~dB}$ SPL and at $110 \mathrm{~dB}$ SPL, presented alone or combined with audio sound. Inter-individual differences of the unpleasantness ratings were further examined with respect to the individual thresholds for the infrasound stimulus (see Sect. 3.4).

Then, ANOVAs were performed with the aligned and ranked data of the numerical scale ratings to investigate effects of the different audio sound stimuli (broadband and sinusoid at $1000 \mathrm{~Hz}$ ) and the levels of both, infrasound and audio sound stimuli, on the unpleasantness ratings, and their interaction. The ART-ANOVA 1 (see Table 2) revealed a significant effect for the factor "level of audio sound" and for the factor "level of infrasound" on the unpleasantness ratings for the sinusoid $12 \mathrm{~Hz}$ at $95 \mathrm{~dB}$ SPL and at $110 \mathrm{~dB}$ SPL, presented alone or combined with audio sound. Moreover, there was a significant interaction between the two factors. Post-hoc pairwise comparisons for the values of the factor "level of audio sound" revealed that there were significant differences between all factor values, except for the comparison between the values "no audio sound" and "low level". The ART-ANOVA 2 (see Table 3) showed significant effects of the factors "type of audio sound", "level of audio sound", and "level of infrasound" on unpleasantness ratings taking into account all audio sound stimuli which were presented alone or along with infrasound. In addition, the ART-ANOVA 2 revealed pairwise significant interactions between the factors "type of audio sound", "level of audio sound", and "level of infrasound", but no significant interaction between all three factors was found. Post-hoc pairwise comparisons for the factor "level of infrasound" revealed that there were only significant differences between the factor value " $110 \mathrm{~dB}$ SPL" compared to the level "95 dB SPL", "80 dB SPL", and "no infrasound".

A closer inspection of the average unpleasantness ratings over the participants calculated as median for each stimulus (Fig. 2) shows that the average unpleasantness for the infrasound stimulus at $95 \mathrm{~dB}$ SPL combined with audio sound at higher levels corresponded closely to that for the same audio sound stimuli presented alone. In addition, these stimuli combinations were on average more 
unpleasant than the isolated infrasound stimulus at $95 \mathrm{~dB}$ SPL. When the infrasound stimulus at $95 \mathrm{~dB}$ SPL was combined with audio sound at a lower level, the average unpleasantness was equal to or slightly higher than the unpleasantness of the same infrasound and audio sound stimulus presented alone.

When the infrasound stimulus at $110 \mathrm{~dB}$ SPL was simultaneously presented with an audio sound stimulus, the unpleasantness of the stimulus combination was on average more unpleasant than the unpleasantness for the same audio sound stimulus presented alone (Fig. 2). In addition, the $12 \mathrm{~Hz}$ stimulus at $110 \mathrm{~dB}$ SPL combined with audio sound was on average equally unpleasant as or, with increasing audio sound level, it was more unpleasant than the same infrasound stimulus presented alone. For example, 13 participants rated the combination of the $12 \mathrm{~Hz}$ stimulus at $110 \mathrm{~dB}$ SPL with the broadband at $53 \mathrm{~dB}$ SPL as more unpleasant than the isolated infrasound stimulus. In addition, 16 participants rated the sinusoid at $1000 \mathrm{~Hz}$ presented at $70 \mathrm{~dB}$ SPL combined with the infrasound stimulus at $110 \mathrm{~dB}$ SPL as more unpleasant than the same infrasound stimulus presented alone, and the remaining three participants rated this combination as equally unpleasant as the isolated infrasound at $110 \mathrm{~dB}$ SPL.

\subsection{Paired comparison task vs numerical scale ratings}

Comparing the distributions of the boxplots in Figures 1 and 2 , it can be seen that both experiments revealed similar results for the unpleasantness for combined infrasound and audio sound stimuli. The results of both unpleasantness rating tasks are plotted against each other in Figure 3. The figure illustrates the high correlation between the results from both rating tasks. The Spearman's rank correlation coefficient between winning rate and numeric scale ratings was significant $\left(r_{p}<0.001\right)$ with correlation coefficients $r_{s}=0.97$ when comparing the arithmetic means of the unpleasantness across the sample for each stimulus, and $r_{s}=0.85$ when comparing the individual data. Both experiments yielded a similar ranking order of the stimuli. By far the most unpleasant stimuli were the sinusoid at $12 \mathrm{~Hz}$ presented at $110 \mathrm{~dB}$ SPL and the sinusoid at $1000 \mathrm{~Hz}$ presented at $70 \mathrm{~dB}$ SPL, either presented alone or simultaneously with another stimulus.

\subsection{Comparison of individual detection thresholds for a sinusoid at $12 \mathrm{~Hz}$ and unpleasantness ratings}

Detection thresholds for a sinusoid at $12 \mathrm{~Hz}$ were measured three times for each participant. The arithmetic mean of the thresholds across the sample was $93.2 \mathrm{~dB}$ $\mathrm{SPL} \pm 1.3 \mathrm{~dB}$ (standard deviation of the mean with $68 \%$ confidence interval). The threshold levels showed a large inter-individual variability with average thresholds between 83 and $104 \mathrm{~dB}$ SPL. Therefore, the $12 \mathrm{~Hz}$ stimulus presented at $95 \mathrm{~dB}$ SPL during the unpleasantness rating task may not have been audible for some of the listeners.

Figure 4 shows the individual unpleasantness ratings for the isolated $12 \mathrm{~Hz}$ stimulus at $95 \mathrm{~dB}$ SPL (upper panel) and

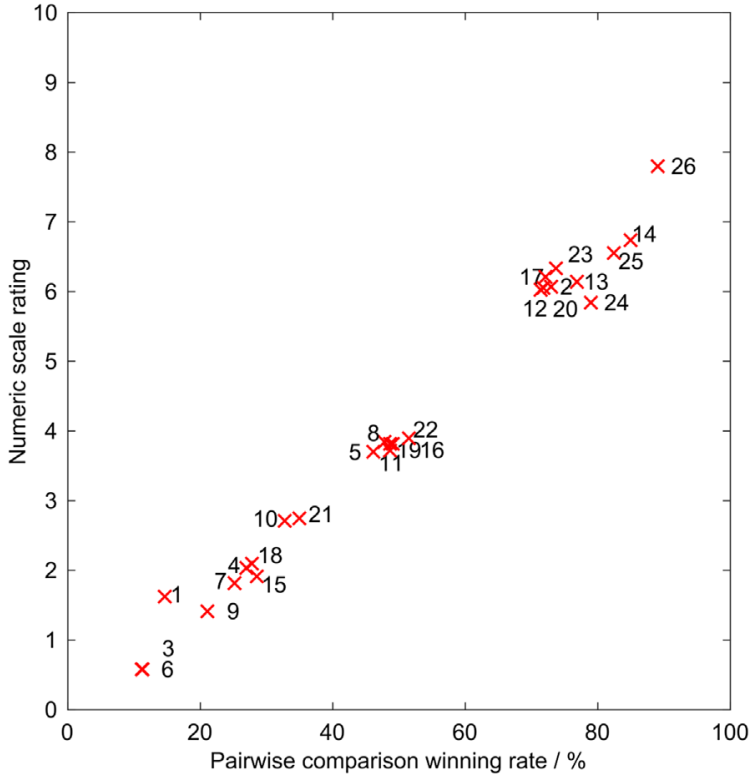

Figure 3. Correlation between average numerical scale rating and the average winning rate $W R$ for each stimulus determined from the results of the pairwise comparison task. The Spearman's rank correlation coefficient between these data was $r_{s}=0.97(p<0.001)$. The list of stimuli including the definition of the stimuli numbers " 1 "-"26" are summarised in Table 1.

$110 \mathrm{~dB}$ SPL (lower panel) using the 11-point numerical scale (see Sect. 2.5.2) as a function of the individual threshold levels for the $12 \mathrm{~Hz}$ stimulus. The figure suggests a relationship between individual threshold level and unpleasantness ratings for the infrasound stimulus at the lower level of $95 \mathrm{~dB}$ SPL, so that participants whose average threshold is below $95 \mathrm{~dB}$ SPL gave higher unpleasantness ratings than the participants with a higher detection threshold. The Spearman's rank correlation coefficients between individual detection thresholds and unpleasantness ratings for the infrasound at $95 \mathrm{~dB}$ SPL, presented alone or combined with audio sound, were calculated using a subset of the data including only the twelve listeners whose average infrasound threshold were below $95 \mathrm{~dB}$ SPL. The correlation was significant only for the broadband stimulus at $21 \mathrm{~dB}$ SPL combined with $12 \mathrm{~Hz}$ at $95 \mathrm{~dB}$ SPL ( $p$-value: $r_{p}=0.021$, correlation coefficient: $\left.r_{s}=-0.653\right)$, and for the broadband stimulus at $36 \mathrm{~dB}$ SPL combined with $12 \mathrm{~Hz}$ at $95 \mathrm{~dB}$ SPL $\left(r_{p}=0.010, r_{s}=-0.711\right)$. The correlation for the isolated $12 \mathrm{~Hz}$ stimulus at $95 \mathrm{~dB}$ SPL was just above significance level of $0.05\left(r_{p}=0.056, r_{s}=-0.564\right)$.

Analysing the unpleasantness ratings for $12 \mathrm{~Hz}$ at $110 \mathrm{~dB}$ SPL, nearly all participants rated the unpleasantness for this stimulus above "4" (see Fig. 4) and the ratings were largely independent of their individual detection thresholds. This was confirmed by a correlation analysis (Spearman's rank correlation) showing that there were no significant correlations $\left(r_{p}>0.05\right)$ comparing individual threshold levels of all 19 listeners to the unpleasantness response on the numerical scale for each stimulus combination including the $12 \mathrm{~Hz}$ stimulus at $110 \mathrm{~dB}$ SPL. 

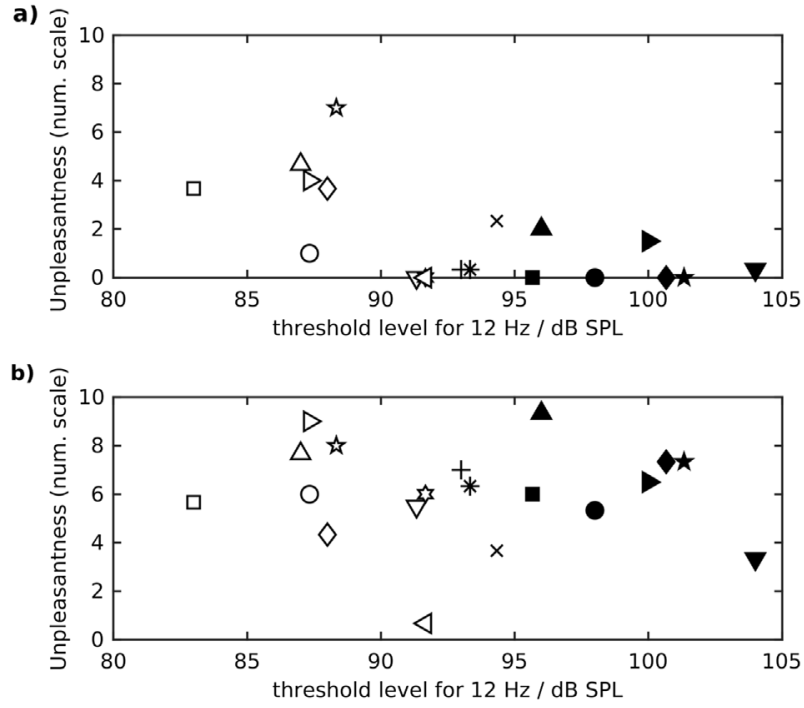

Figure 4. Unpleasantness ratings for the sinusoid at $12 \mathrm{~Hz}$ in isolation presented at $95 \mathrm{~dB}$ SPL (upper plot (a)) and at $110 \mathrm{~dB}$ SPL (lower plot (b)) in dependence on individual detection threshold levels for the sinusoid at $12 \mathrm{~Hz}$ of 19 listeners. Each marker indicates the average unpleasantness rating and average detection threshold of one listener.

\section{Discussion}

The main findings of this study are that the levels of infrasound and audio sound stimuli, and the type of audio sound stimulus (broadband vs. sinusoid) have significant effects on unpleasantness ratings for combined infrasound and audio sound stimuli. Moreover, combinations of infrasound and audio sound were rated as equally or more unpleasant than either sound presented alone. Particularly high inter-individual differences were found for psychoacoustic variables including infrasound as in other studies (e.g. [4, 5, 31]). The unpleasantness ratings using the numerical scale can only in part be related to the individual detection threshold for infrasound. The results of both rating methods (paired comparison task and rating task using the 11-point-numerical scale) were similar so that they are mainly discussed together in the following.

\subsection{Isolated infrasound}

This study showed that the isolated $12 \mathrm{~Hz}$ stimulus at $110 \mathrm{~dB}$ SPL, that is a sound pressure level clearly above the individual threshold levels, resulted in a high unpleasantness similar to the unpleasantness related to the sinusoid at $1000 \mathrm{~Hz}$ presented at $70 \mathrm{~dB}$ SPL. Interpolating the data of Møller's study [8] - rating the annoyance rather than the unpleasantness - a level of around 113-118 dB SPL (depending on the rating method and exposure time) is needed for a sinusoid at $12 \mathrm{~Hz}$ to be equally annoying as a sinusoid at $1000 \mathrm{~Hz}$ presented at $70 \mathrm{~dB}$ SPL. This level is very close to the infrasound level of $110 \mathrm{~dB}$ SPL applied in this study.

In general, a high increase in unpleasantness was found comparing the unpleasantness for the $12 \mathrm{~Hz}$ stimulus at
$95 \mathrm{~dB}$ SPL and $110 \mathrm{~dB}$ SPL which is much steeper than the increase with sound pressure level in unpleasantness for the audio sound stimuli. Similar results are found for measurements of equal-annoyance [7, 8], equal-loudness [4, 6], and equal-unpleasantness curves [9] showing that distances between all of these curves are significantly smaller in the infrasound than in the audio-sound frequency range.

Comparing the average unpleasantness and estimated loudness of the stimuli it can be observed that the sinusoid at $12 \mathrm{~Hz}$ at $110 \mathrm{~dB}$ SPL which has a loudness around $40-50$ phon according to [4] is more unpleasant than the audio sound stimuli at 20 phon and at 50 phon. Moreover, this infrasound stimulus is more unpleasant than the broadband stimulus at 70 phon and it has about the same unpleasantness as the $1000 \mathrm{~Hz}$ stimulus at 70 phon. This indicates that the unpleasantness cannot be derived directly from the perceived loudness which is in line with the results of Behler and Uppenkamp [31]. In addition, it is important to keep in mind that ratings of the unpleasantness of infrasound as a function of the perceived loudness can vary considerably across individuals [31].

There were large inter-individual differences in the unpleasant ratings of isolated infrasound. However, these inter-individual differences were considerably greater than those of the ratings of isolated audio sound only in the procedure using the numerical scale. The inter-individual differences in the unpleasantness rating of the applied stimuli differed between the paired comparison experiment and the rating task with the numerical scale. This might be due to the different tasks and quantities determined, i.e. winning rate and numerical scale rating. Inter-individual differences of the unpleasantness ratings for the isolated infrasound using the numerical scale can in part be linked to the individual threshold level: For participants with thresholds below $95 \mathrm{~dB}$ SPL, the correlation between the individual threshold level and the unpleasantness ratings for the sinusoid at $95 \mathrm{~dB}$ SPL was just slightly higher than the significance level. This indicates that the unpleasantness for infrasound at a level slightly above detection threshold is related to the individual threshold level. On the contrary, when the infrasound level is increased, the unpleasantness ratings for these stimuli are not any more predictable by the individual detection thresholds. Therefore, additional important factors may be a reason for the observed inter-individual differences in unpleasantness for the infrasonic stimulus at $110 \mathrm{~dB}$ SPL. Differences in general behaviour of the participants may be the reason why some participants rated the stimuli as more unpleasant on the numerical scale than others. This could be caused by individual differences in noise sensitivity. However, the assessment of noise sensitivity was not part of this study and would require different experimental tools like a noise sensitivity questionnaire (e.g. [32, 33]).

\subsection{Infrasound combined with audio sound}

Audio sound stimuli combined with subthreshold infrasound (12 Hz at $80 \mathrm{~dB}$ SPL) were rated as equally unpleasant as isolated audio sounds. This is another indication that 
the detection threshold is also in the infrasound frequency range an important parameter for characterisation of the impact of sound on humans.

The unpleasantness for infrasound presented around average threshold level $(12 \mathrm{~Hz}$ at $95 \mathrm{~dB}$ SPL) combined with audio sound was also on average rated as equally unpleasant as isolated audio sound. A possible reason for this is that audio sound can mask infrasound [15], when audio sound is presented at a sufficient level. Due to this masking effect, an audio sound stimulus presented alone or in combination with a $12 \mathrm{~Hz}$ stimulus at $95 \mathrm{~dB}$ SPL may result in a similar auditory sensation and, thus, also result in the same perceived unpleasantness. Another possible reason is that some of the participants with high infrasound thresholds had not perceived the infrasound stimulus presented at $95 \mathrm{~dB}$ SPL at all. In addition, there were large inter-individual differences for the rating of the sinusoid at $95 \mathrm{~dB}$ SPL combined with audio sound which might be due to the large variation of the individual threshold levels.

This study revealed that the infrasound presented well above-threshold level (12 $\mathrm{Hz}$ at $110 \mathrm{~dB}$ SPL) combined with audio sound is perceived as more unpleasant than isolated audio sound so that the infrasound stimuli dominate the perceived unpleasantness. In the rating task using the numerical scale, it was observed that the unpleasantness for a combined stimulation for infrasound above-threshold level and audio sound is rated on average as either equally or more unpleasant than isolated infrasound (see Sect. 3.2). In the paired comparison task (see Sect. 3.1), an increase in unpleasantness was found for the stimulus No. 26 (combination of sinusoid at $12 \mathrm{~Hz}$ presented at $110 \mathrm{~dB}$ SPL and sinusoid at $1000 \mathrm{~Hz}$ presented at $70 \mathrm{~dB} \mathrm{SPL}$ ) compared to stimulus No. 2 (sinusoid at $12 \mathrm{~Hz}$ presented at $110 \mathrm{~dB}$ SPL). A possible reason for this might be that a modulation effect $[13,14]$ of audio sound caused by the infrasound may increase the unpleasantness compared to infrasound presented alone. This modulation effect may be more perceptible for a pure tone as audio sound stimuli.

Comparing these results of our study with the results of Møller's study [8], both revealed that combinations of infrasound and audio sound are rated as equal, or slightly more, annoying/unpleasant than the most annoying/unpleasant isolated stimuli. However, it has to be taken into account that different measurement procedures, exposure times and stimuli were applied in both studies. Furthermore, a monaural presentation by an insert-earphone sound source system was used in our study instead of a whole-body pressure-field chamber like in [8]. However, since these differences did not change the overall outcome of the studies the hearing sensation probably dominates over a potential perception via the whole-body stimulation.

\subsection{Comparison of results of the paired comparison task and of the 11-point-numerical ratings task}

The results of both measurement procedures, the 11-point-numerical scale rating task and the pairwise comparison task, were consistent. In addition, both unpleasantness rating tasks yielded a similar rank order of the unpleasantness for the investigated stimuli. Both methods are suitable to investigate the unpleasantness for combined infrasound and audio sound stimuli. However, the advantage of the paired comparison task is that it is easier for the listeners to perform a relative comparison of two stimuli with respect to their unpleasantness, rather than to judge a single stimulus on a predefined scale. On the other hand, the paired comparison task is considerably more time consuming than the numerical scale experiment.

It has to be pointed out that in this study the numeric scale rating task was performed after the paired comparison task and, therefore, the participants had already known and perceived the set of the applied stimuli prior to the numerical rating tasks. If the numerical rating task was performed as isolated experiment, a trainings phase should be added at the beginning of the experiment.

\subsection{Limitations of this study}

Within this study the stimulus duration was limited to two seconds to keep the overall time needed to complete the experiment within an acceptable limit for the participants. Therefore, conclusions about the effects of long-time exposure to infrasound combined with audio sound cannot be drawn based on the data in this study. Furthermore, this study was limited to the perception of infrasound by means of the auditory system by transmitting the sound stimuli directly into the ear canal of the participants. Other sensation pathways can come along with the sound exposure to environmental infrasound noise, like tactile sensation (vibrations caused by, e.g., road and railway traffic) or visual sensation (e.g. driving cars in the case of road traffic noise, moving turbine blades in the case of wind turbine noise). These sensation mechanisms and other factors like, e.g., individual noise sensitivity of the participants could not be considered in this study, but they could still affect the perceived annoyance related to environmental infrasound noise.

The results of the experiments could be biased because the informed consent paperwork handed out to participants before the experiments mentioned that the study aims to investigate the perception of infrasound. However, the experimental supervisor avoided using the term "infrasound" during the oral instructions before starting the experiments and within the breaks in order to keep the risk of bias low.

\section{Conclusion}

The main findings of this study can be summarized as follows:

1. Audio sound presented alone and in combination with subthreshold infrasound resulted in the same unpleasantness, which may implicate that environmental noise with inaudible infrasound components is not perceived as significantly more unpleasant than noise with no infrasound components. 
2. The study showed the tendency that the unpleasantness for infrasound presented at a level only slightly above the average threshold level is related to the individual detection threshold for infrasound, whereas for an increased level of the infrasound the perceived unpleasantness cannot be predicted any more by the individual threshold levels. Together with conclusion (1), this shows that the detection threshold is an important parameter for the estimation of annoyance also in the infrasound frequency range.

3. Combinations of infrasound and audio sound, both presented at a sufficient level above the individual threshold, can be more unpleasant than the same infrasound and audio sound stimuli presented alone. Infrasound can then become a dominating factor.

\section{Conflict of interest}

Author declared no conflict of interests.

\section{Acknowledgments}

This study is part of the Ears II project "Metrology for modern hearing assessment and protecting public health from emerging noise source". This project has received funding from the EMPIR programme co-financed by the Participating States and from the European Union's Horizon 2020 research and innovation programme. The authors would like to express their gratitude to all participants of this study. Furthermore, the authors would like to thank Euginia P. Stederi for supporting the implementation and the conduction of the experiments and Kirsten Netter for providing MATLAB scripts for the paired comparison experiment. We would also like to thank our colleagues at PTB and at University of Oldenburg supporting this study, especially Oliver Behler, Marion Bug, and Sven Vollbort. The helpful comments and suggestions by three anonymous reviewers are gratefully acknowledged.

\section{References}

1. B. Berglund, P. Hassmén, R.F.S. Job: Sources and effects of low-frequency noise. The Journal of the Acoustical Society of America 99, 5 (1996) 2985-3002.

2. H.G. Leventhall: Low frequency noise and annoyance. Noise and Health 6, 23 (2004) 59-72.

3. C. Baliatsas, I. van Kamp, R. van Poll, J. Yzermans: Health effects from low-frequency noise and infrasound in the general population: Is it time to listen? A systematic review of observational studies. Science of the Total Environment 557-558 (2016) 163-169.

4.H. Møller, C.S. Pedersen: Hearing at low and infrasonic frequencies. Noise and Health 6, 23 (2004) 37-57.

5. R. Kühler, T. Fedtke, J. Hensel: Infrasonic and low-frequency insert earphone hearing threshold. The Journal of the Acoustical Society of America 137 (2015) EL347-EL353.
6. R. Kühler, J. Hensel, C. Koch, M. Bauer, T. SanderThömmes: Auditory cortex activation by infrasonic and low-frequency sound of equalized individual loudness, in Proceedings of Euronoise, Maastricht, the Netherlands. 2015, pp. 2577-2582.

7. J. Andresen, H. Møller: Equal annoyance contours for infrasonic frequencies. Journal of Low Frequency Noise, Vibration and Active Control 3, 3 (1984) 1-9.

8. H. Møller: Annoyance of audible infrasound. Journal of Low Frequency Noise, Vibration and Active Control 6, 1 (1987) $1-17$.

9. Y. Inukai, N. Nakamura, H. Taya: Unpleasantness and acceptable limits of low frequency sound. Journal of Low Frequency Noise, Vibration and Active Control 19, 3 (2000) $135-140$.

10. R. Guski, U. Felscher-Suhr, R. Schuemer: The concept of noise annoyance: How international experts see it. Journal of Sound and Vibration 223, 4 (1999) 513-527.

11. R. Guski: Personal and social variables as codeterminants of noise annoyance. Noise and Health 1, 3 (1999) 45-56.

12.R. Guski: Psychological methods for evaluating sound quality and assessing acoustic information. Acta Acustica United With Acustica 83 (1997) 765-774.

13. T. Marquardt, C. Jurado: Amplitude modulation may be confused with infrasound. Acta Acustica United With Acustica 104 (2018) 825-829.

14. N. Salt, J.T. Lichtenhan, R.M. Gill, J.J. Hartsock: Large endolymphatic potentials from low-frequency and infrasonic tones in the guinea pig. The Journal of the Acoustical Society of America 133, 3 (2013) 1561-1571.

15. E. Burke, J. Hensel, T. Fedtke, S. Uppenkamp, C. Koch: Detection thresholds for combined infrasound and audiofrequency stimuli. Acta Acustica United With Acustica 105, 6 (2019) 1173-1182.

16. E. Burke, J. Hensel: Sound source system for investigating the auditory perception of infrasound accompanied by audio sound. Acta Acustica United With Acustica 105, 5 (2019) 869-874.

17. J.S. Bradley: Annoyance caused by constant-amplitude and amplitude-modulated sounds containing rumble. Noise Control Engineering Journal 42 (1994) 203-208.

18. B. Schäffer, R. Pieren, S.J. Schlittmeier, M. Brink: Effects of different spectral shapes and amplitude modulation of broadband noise on annoyance reactions in a controlled listening experiment. International Journal of Environmental Research and Public Health 15, 5 (2018) 1-17.

19. ISO 8253-1: Acoustics - Audiometric Test Methods - Part 1: Pure-tone Air and Bone Conduction Audiometry, 2010.

20. K. Zimmer, W. Ellermeier, C. Schmid: Using probabilistic choice models to investigate auditory unpleasantness. Acta Acustica United With Acustica 90 (2004) 1019-1028.

21. J.M. Fields, R.G. De Jong, T. Gjestland, I.H. Flindell, R.F.S. Job, S. Kurra, P. Lercher, M. Vallet, T. Yano, R. Guski, U. Felscher-Suhr, R. Schumer: Standardized general-purpose noise reaction questions for community noise surveys: research and recommendation. Journal of Sound and Vibration 242, 4 (2001) 641-679.

22. ISO/TS 15666: Acoustics - Assessment of Noise Annoyance by Means of Social and Socio-Acoustic Surveys, 2003.

23. E. Burke, E.P. Stederi, S. Uppenkamp, C. Koch: Investigation of the unpleasantness of infrasound combined with audio sound using psychoacoustic scaling methods, in Proceedings of the 23rd International Congress on Acoustics, Aachen, Germany. 2019, pp. 3000-3006.

24. IEC 60318-4, Electroacoustics - Simulators of Human Head and Ear - Part 4 - Occluded-Ear Simulator for the 
Measurement of Earphones Coupled, to the Ear by Means of Ear Inserts, Geneva, Switzerland, 2010.

25. DIN 45681:2005-03: Akustik - Bestimmung der Tonhaltigkeit von Geräuschen und Ermittlung eines Tonzuschlages für die Beurteilung von Geräuschimmissionen (Acoustics Determination of Tonal Components of Noise and Determination of a Tone Adjustment for the Assessment of Noise Immissions), 2005.

26. R.P. Hellman: Loudness, annoyance, and noisiness produced by single-tone-noise complexes. The Journal of the Acoustical Society of America 72, 1 (1982) 62-73.

27. ANSI S 3.4-2007: Procedure for the Computation of Loudness of Steady Sounds, 2007.

28. J.O. Wobbrock, L. Findlater, D. Gergle, J.J. Higgins: The aligned rank transform for nonparametric factorial analyses using only ANOVA procedures, in Proceedings of the Annual Conference on Human Factors in Computing Systems (CHI '11), Vancouver, Canada. 2011, pp. 143-146.
29. M. Kay, J.O. Wobbrock: ARTool: Aligned Rank Transform for Nonparametric Factorial ANOVAs. R package version 0.10.6, 2019.

30. S.D. Ewert: AFC - A modular framework for running psychoacoustic experiments and computational perception models, in Proceedings of the International Conference on Acoustics AIA-DAGA 2013, Merano, Italy. 2013, pp. 13261329 .

31. O. Behler, S. Uppenkamp: Activation in human auditory cortex in relation to the loudness and unpleasantness of lowfrequency and infrasound stimuli. PLoS One 15, 2 (2020) $1-20$.

32. M. Schutte, A. Marks, E. Wenning, B. Griefahn: The development of the noise sensitivity questionnaire. Noise and Health 9, 34 (2007) 15-24.

33. N.D. Weinstein: Individual differences in reactions to noise: A longitudinal study in a college dormitory. Journal of Applied Psychology 63, 4 (1978) 458-466.

Cite this article as: Burke E, Uppenkamp S \& Koch C. 2020. A psychoacoustical study to investigate the perceived unpleasantness of infrasound combined with audio-frequency sound. Acta Acustica, 4, 20. 